\title{
Strain Rate Dependent Constitutive Modelling of 3D Printed Polymers
}

\author{
Maria Lißner ${ }^{1 *}$, Daniel Thomson ${ }^{1}$, Nik Petrinic ${ }^{1}$, and Jeroen Bergmann ${ }^{1}$ \\ ${ }^{1}$ Department of Engineering Science, University of Oxford, Parks Road, Oxford OX1 3PJ
}

\begin{abstract}
Experimental results from 3D printed TPC (thermoplastic copolyester) compression specimens were used to develop a combined experimental-numerical framework to support the design of e.g. 3D printed mouthguards. First, a commercially available material model capable of representing the strain-rate dependent behaviour of materials undergoing large deformations is identified. Second, experimental results from solid 3D printed compression specimens are used to calibrate the identified material models. Third, 3D printed compression specimens with two different cavity geometries are used to assess the ability of the material model to accurately reproduce the experimental observations. The numerical investigation indicates a good representation of the strain rate dependent experimental results of 3D printed specimens.
\end{abstract}

\section{Introduction}

Polymeric soft materials are widely used for protection in many sports, e.g. as mouthguards in boxing or as liners in hockey helmets. This is due to their advantageous material properties such as high energy dissipation, low density and low weight. Nevertheless, providing protection from potentially life-threatening impacts in contact sports (e.g., boxing, rugby) or sports with hard ball contact (e.g., field hockey, cricket) can be extremely challenging and requires continuous innovation and improvement of existing designs. In addition, many other types of potentially debilitating head injuries, such as sport related concussion, intra cranial bleeding (ICB), and traumatic brain injury (TBI) cannot be fully prevented even if protective equipment such as helmets or mouthguards are already in place [1]. Some of these can be attributed to the inability of sports equipment to proactively inform the athlete about their health management.

To address this challenge, wearable sensor technologies (i.e. GPS, or heart monitoring systems) are increasingly being considered to monitor the health performance of athletes during competition $[2,3]$. Nevertheless, their positioning is of high importance in order to measure the performance of interest as accurately as possible while remaining mechanically sound. The integration of sensors into protective equipment provides a suitable approach to meet these requirements [4]. Additive manufacturing technology is a potential tool that can help achieve this: Cavities to embed the sensors can be printed precisely while minimising

* Corresponding author: maria.lissner@eng.ox.ac.uk 
excess material. However, the 3D printed safety devices still need to perform reliably under demanding mechanical loads - the impact protection of these protective systems must not be compromised. This demands a fundamental understanding of the mechanical performance of the devices, which must be resistant to impact scenarios and at the same time be able to accommodate sensor systems.

The aim of this work is to develop a numerical-experimental framework that enables the reliable design of smart sports protection devices (such as mouthguards). First, a commercially available material model is identified that is able to represent the experimentally observed strain rate dependent response of the materials. Second, experimental results of 3D printed solid compression specimens are used to calibrate the identified material model. Third, 3D printed compression specimens with two different cavity geometries are used to evaluate the ability of the calibrated material model to accurately represent the experimental observations. Finally, the results of the numerical investigations are discussed in terms of their potential for reliable predictive design modelling of larger scale applications.

\section{Material and Methods}

A thermoplastic material was tested at different strain rates using different topologies. The compression tests were conducted using the Split Hopkinson Pressure Bar and Instron machine setup. The numerical investigation is based on this experimental campaign using the finite element software ABAQUS/Explicit. Experimental results from solid compression specimens are used to calibrate material models suitable to represent both the observed large strain and the non-linear strain rate dependence. The calibrated material model is then verified by modelling the other two configurations (cubic and spherical topologies).

\subsection{Material and specimen designs}

The investigated 3D printable material Arnitel ${ }^{\circledR}$ ID 2045 Natural (DSM, The Netherlands) is a TPC (Thermoplastic Copolyester) that is highly flexible and has excellent energy return suitable for sports protective equipment.

The compression specimens have a diameter of $12 \mathrm{~mm}$ and a thickness of $4 \mathrm{~mm}$ which is similar to the thickness of mouthguards. The air cells for the two different topology structures are $3 \times 2 \times 2 \mathrm{~mm}$ and $\varnothing 2 \mathrm{~mm}$ for the cubic and spherical topology, respectively. The wall thickness is $1 \mathrm{~mm}$ in both cases. Figure 1(a) illustrates the different specimen configurations.

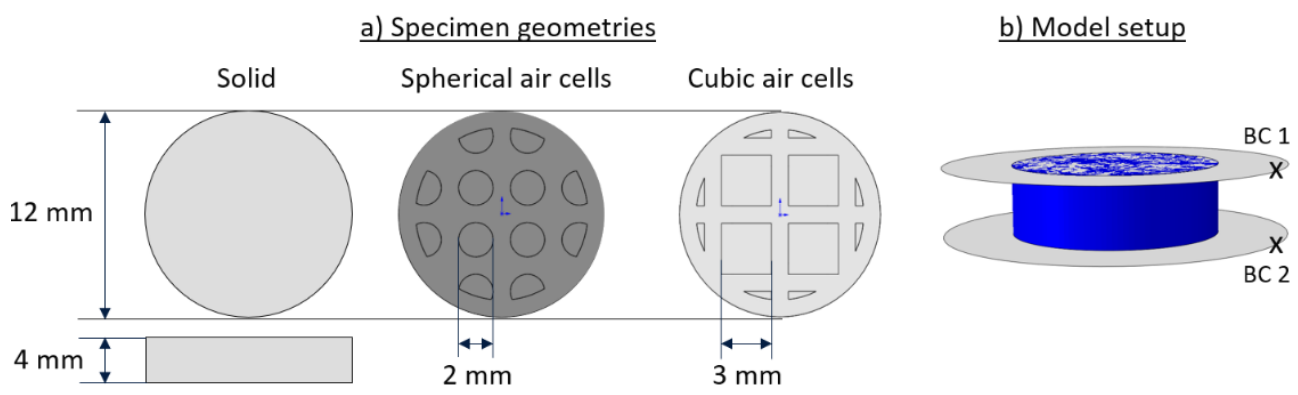

Figure 1. Graphical illustration of a) the specimen geometries with different topologies and b) the model setup. 


\subsection{Material model}

\subsubsection{Parallel Rheological Framework (PRF)}

The non-linear strain rate behaviour of the investigated material can be described with the Parallel Rheological Framework (PRF) introduced by Hurtado et al. [5,6]. This framework allows the parallel superposition of viscoelastic and elastic networks. The deformation gradient of each network $i$ for finite deformation in elastic materials is assumed as follows $[5,6]$

$$
\begin{aligned}
\boldsymbol{F} & =\boldsymbol{F}_{i}^{e} \boldsymbol{F}_{i}^{c r} \quad i=1 \ldots N \\
\boldsymbol{F} & =\boldsymbol{F}_{0}^{e}
\end{aligned}
$$

where $\boldsymbol{F}_{i}^{e}$ represents the elastic part of the deformation gradient and $\boldsymbol{F}_{i}^{c r}$ represents the creep part of the deformation gradient in the viscoelastic networks. $\boldsymbol{F}_{0}^{e}$ is the elastic component of the equilibrium network. Isotropic behaviour and incompressibility are assumed $[5,6]$.

\subsubsection{Elastic Behaviour}

The elastic response of the networks is described with a hyperelastic strain energy potential using the principle of virtual work. It is assumed that all networks are described by the same strain energy potential formulation combined with the assumption that the total strain energy $\mathbf{U}_{\mathrm{T}}$ is equal to the weighted sum of the strain energies of all the networks as

$$
\boldsymbol{U}_{T}=\sum_{i=0}^{N} S_{i} U\left(\boldsymbol{C}_{i}^{e}\right)
$$

where $s_{i}$ is the stiffness ratio of the ith network, representing the volume fraction of the network and $\boldsymbol{C}_{i}^{e}=\left(\boldsymbol{F}_{i}^{e}\right)^{T} \boldsymbol{F}_{i}^{e}$ is the elastic right Cauchy-Green tensor in each network. The stiffness ratios as material parameters are required to satisfy the relation

$$
\sum_{i=0}^{N} S_{i}=1 .
$$

The hyperelastic material behaviour is modelled with the Neo Hooke model which is related solely to the first Cauchy strain tensor invariant $\mathbf{I}_{1}$ as follows

$$
\boldsymbol{U}_{N H}=C_{1}\left(\boldsymbol{I}_{1}-3\right)
$$

where $C_{I}$ is the material parameter and the first invariant $\mathbf{I}_{1}$ is represented by the primary elongations $\lambda_{i}(\mathrm{i}=1,2,3)$ as

$$
\boldsymbol{I}_{1}=\lambda_{1}^{2}+\lambda_{2}^{2}+\lambda_{3}^{2}
$$

The principal stress $\sigma$ is calculated using the following relationship between the strain energy and the elongation

$$
\sigma_{i}=\lambda_{i} \frac{\partial U_{N H}}{\partial \lambda_{i}}-p, i=1,2,3
$$

where $p$ is the hydrostatic pressure and obtained through the kinetic boundary conditions, which are for the considered uniaxial case as follows

$$
\begin{aligned}
& \sigma_{1}=\sigma, \sigma_{2}=\sigma_{3}=0 \\
& \lambda_{1}=\lambda, \lambda_{2}=\lambda_{3}=\lambda^{-1 / 2} .
\end{aligned}
$$

The hydrostatic pressure $p$ can then be calculated by substituting $\sigma_{2}=0$ into Eq. (6) alongside with Eq. (4), which results in 


$$
p=\lambda_{2} \frac{\partial I_{1}}{\partial \lambda_{2}} C_{1}
$$

Substituting Eq. (4) and (8) into Eq. (6) leads to the calculation of $\sigma_{l}$ as

$$
\sigma_{1}=\left(\lambda_{1} \frac{\partial I_{1}}{\partial \lambda_{1}}-\lambda_{2} \frac{\partial I_{1}}{\partial \lambda_{2}}\right) C_{1}
$$

With the relationships for the uniaxial case in Eq. (7) the calculation of the uniaxial hyperelastic principal stress is as follows

$$
\sigma=2 C_{1}\left(\lambda-\lambda^{-2}\right) \text {. }
$$

\subsubsection{Viscous Behaviour}

The viscoelastic behaviour of the material is defined by the strain hardening power law described in $[6,7]$ and supported in ABAQUS following

$$
\dot{\varepsilon}^{c r}=\left\{A q^{n}\left[(m+1) \varepsilon^{c r}\right]^{m}\right\}^{\frac{1}{m+1}}
$$

where $\mathrm{A}, \mathrm{m}$ and $\mathrm{n}$ are material parameters.

\subsubsection{Parameter Identification}

Parameter identification was performed using the nonlinear least square algorithm lsqnonlin in MATLAB. First, the hyperelastic material model was calibrated using the results for the low-rate solid compression specimens. Second, the strain rate dependent material parameters were determined using the experimental results of the solid compression specimens for all three loading rates simultaneously. To use the strain hardening potential law in ABAQUS, the parameters $m$ and $n$ are defined to be in the ranges $[-1,0]$ and $[0,10]$, respectively. Finally, a most adequate set of material parameters was determined that best fits the strain rate dependent experimental results.

\subsubsection{Model setup}

The identified material parameters are used to numerically model the strain rate-dependent hyperelastic material behaviour of 3D-printed compression specimens. For this purpose, the geometries for the three different specimen configurations were modelled in the finite element (FE) software ABAQUS/Explicit. Similar to the experiments, the specimens were placed between two bars, which were modelled as rigid bodies due to their high stiffness compared to the stiffness of the TPC. The contact definition between the specimens and the bars is defined as frictionless. The lower rigid bar is fixed (BC 2), while the upper bar is loaded with the equivalent displacement curves of the experiments for up to $45 \%$ true strain (BC 1). Figure 1(b) represents the set-up graphically.

Six-node tetrahedral elements are used with a mesh size of $0.25 \mathrm{~mm}$. The constitutive parameters describing the hyperelastic and strain rate dependent material behaviour are implemented into the FE for the two networks - hyperelastic (Neo Hooke model) and viscoelastic (Strain hardening power law). The true stress-true strain response is obtained at the applied load position (BC 1). 


\section{Results and Discussion}

The suitability of the identified parameters is evaluated by numerical modelling of the solid 3D printed compression specimens. The experimental results of 3D printed specimens with different cellular structures are used to verify the calibrated material model and to assess the suitability of the performed modelling strategy for 3D printed thermoelastic materials.

\subsection{Strain-rate dependent material parameter identification}

Table 1 presents the obtained parameters for the Neo Hooke material and the strain hardening power law. Figure 2(a) shows the numerical prediction of the experimentally obtained strain rate dependent material behaviour of solid compression specimens. The root mean square errors (RMSE) between the experimental (averaged) and numerical representation for the different strain rates are highlighted in Figure 2(a). This demonstrates that the Neo Hooke model is a simple and suitable tool in combination with the strain hardening power law for modelling the strain rate dependent behaviour of the solid TPC material. Nevertheless, due to its nature, the stress-strain relationship is represented as mostly linear, which can have a negative impact on the correct prediction of the behaviour, depending on the application.

Table 1. Identified parameters for numerical simulation

\begin{tabular}{ll|llll}
\multicolumn{2}{r|}{ Neo Hooke model } & \multicolumn{4}{c}{ Strain hardening power law } \\
\hline C & D & A & $\mathrm{n}$ & $\mathrm{M}$ & sratio \\
2.1636 & 0.0000 & 0.4245 & 0.9964 & -0.5 & 0.9
\end{tabular}
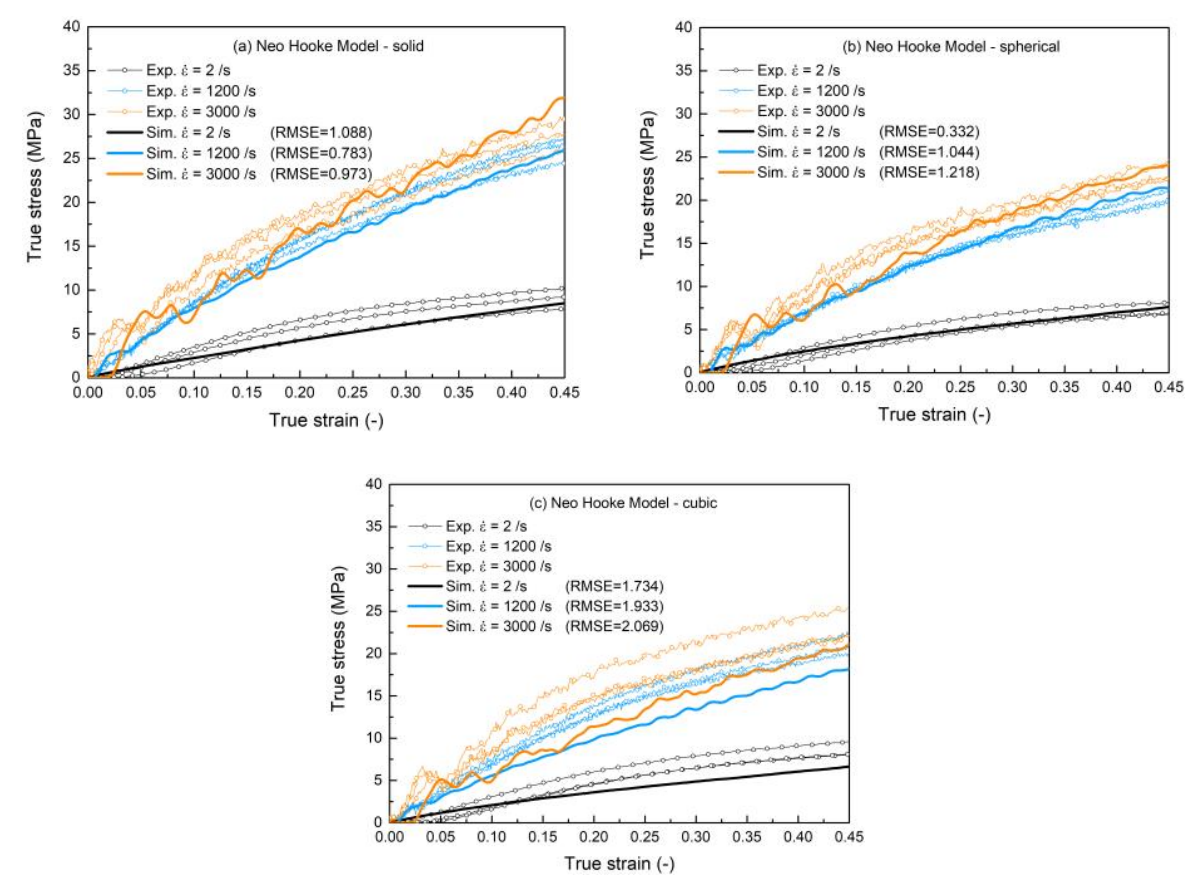

Figure 2. Strain-rate dependent true stress - true strain experimental and numerical results for compression specimens with a) solid, b) spherical air cells and c) cubic air cells. 


\subsection{Model verification}

Figure 2(b) and (c) show the numerical representation of the experimental results with the errors for the cubic and spherical cell structures, respectively. The experimental results for the cubic air cells are slightly underrepresented by the model when compared to the results of the spherical air cells. This difference might be two folded: on one hand, the 3D printing process might have introduced inhomogeneities and on the other hand, the cubic geometry resulted in sharp edges which are most likely the source of stress concentrations. Both reasons, might result in this larger discrepancy of the numerical representation.

\section{Conclusion}

The main objective of this study was to demonstrate the suitability of calibrating the strain rate dependency of a commercially available hyperelastic material model using 3D printed solid specimens experiments to be used to predict the material behaviour of $3 \mathrm{D}$ printed structures with different topologies. The following conclusions can be drawn from this research:

1. The Neo-Hooke model and strain hardening power law incorporated in ABAQUS were successfully calibrated using strain rate dependent experimental results of $3 \mathrm{D}$ printed solid compression specimens.

2. The calibrated strain rate dependent constitutive relationship using the PRF was successfully verified by predicting the experimental response of strain-rate dependent experiments of 3D-printed printed specimens with different cellular structures (cubic and spherical).

3. The presented modelling strategy provides a useful tool when predicting the mechanical performance of different 3D printed topologies during the design process of larger applications, such as mouthguards.

\section{References}

1. D.H. Daneshvar, C.M. Baugh, C.J. Nowinski, A.C. McKee, R.A. Stern, R.C. Cantu, Helmets and Mouth Guards: The Role of Personal Equipment in Preventing SportRelated Concussions. Clinics in Sports Medicine, 30(1), 145-163, (2011)

2. M. Higgins, P.D. Halstead, L. Snyder-Mackler, D. Barlow, Measurement of impact acceleration: Mouthpiece accelerometer versus helmet accelerometer. J. Athl. Train., 42(1), 5-10, (2007)

3. W. Davies, H. Ye, J. Bergmann, Unobtrusive bioanalytics for impact-related sport activities. Advances in Intelligent Systems and Computing, 792, 285-93 (2019)

4. Bridgman, Helen, Man Ting Kwong, and Jeroen HM Bergmann. Mechanical safety of embedded electronics for in-body wearables: A smart mouthguard study. Annals of biomedical engineering 47.8 (2019): 1725-1737.

5. J.A. Hurtado, I. Lapczyk, S.M. Govindarajan, Parallel Rheological Framework to model non-linear viscoelasticity, permanent set and Mullins effect in elastomers, Constitutive Models for Rubber VIII, 95-100 (2013)

6. I. Lapczyk, J.A. Hurtado, S.M. Govindarajan, A parallel rheological framework for modelling elastomers and polymers, $182^{\text {nd }}$ Technical Meeting of the Rubber Division of the American Chemical Society, 1840-1859, (2012) 